\title{
LOS ÓRGANOS DE LA CATEDRAL DE VALENCIA EN EL SIGLO XVII
}

Desde que Mn. Anglés publicara en 1927 el primer volumen de la Opera Omnia de Juan Cabanilles, el interés por conocer la realidad de aquellos instrumentos con los que contaba el organista de la catedral valentina ha ido siempre in crescendo. Parece que los hados no han sido favorables. Primero fue el testamento de Cabanilles que no aparece en el notario y fecha indicados en su acta de defunción. Luego, el contrato de construcción del órgano que presidió todo el XVII valenciano, tampoco ha sido localizado hasta ahora. Ciertamente no consta en los lugares que, lógicamente, debiera estar. Suponemos que aparecerá en los sitios más insospechados.

Mn. Anglés, que en la recopilación de datos de los archivos catedralicios valentinos, siempre fue de la mano del canónigo archivero, D. José Sanchis Sivera y de D. Vicente Ripollés, queriendo contribuir a vislumbrar el órgano de la catedral de forma que colaborara a una mejor intelección de la obra de Cabanilles, nos dio a conocer el órgano de la Catedral de Lérida para hacer un parangón entre los instrumentos de las dos catedrales por ser ambos del mismo constructor y realizado, el de Valencia, a los pocos años de hecho el de Lérida (1624-1632).

El mismo Anglés decía que «sería cosa muy interesante para nosotros el saber a ciencia fija la contextura del órgano que Cabanilles tocó en la Catedral de Valencia durante cuarenta y siete años»*.

Hoy, con los dos documentos que se aportan, no solo conocemos, casi en su totalidad, la composición de los órganos que tuvo la Catedral en tiempos de Cabanilles, sinotambién la forma de proceder en la formación de diversas combinaciones empleadas, ordinariamente, en la interpretación de las formas orgánicas clásicas del momento; y la podemos conocer de la mano del constructor mismo, o sea con palabras de Fray Antonio Llorens y Fray Juan Olius, su ayudante.

* Anglés, H.: Musici organici Johannis Cabanilles, Opera Omnia. (Barcelona, 1927). Vol. I, XXXIII. 
Quedan todavía cabos sueltos, pero, en líneas generales, podemos conocer la composición del órgano «gran» de la Catedral valentina.

Que la composición de los dos órganos catedralicios de Valencia que pretendemos presentar es la misma que conoció Cabanilles, no se presta, creo, a ponerlo en tela de juicio, puesto que no conocemos ninguna otra restauración-reordenación realizada en el siglo XVII, y, además, conociendo la idiosincrasia de la Cabildos Capitulares, pese a que eran años de abundancia si los comparamos con los actuales, es impensable imaginar dos reestructuraciones en menos de cuarenta años. Tendría que haber alguna razón especial para ello, y, que se sepa, no existe tal razón especial.

En estos dos documentos se plantea directamente la forma de registrar, pero no en abstracto, sino concretamente, en el instrumento del que se puede disponer, con lo cual el organero se ve obligado a presentarnos la registración con los juegos que cuenta.

Uno de los problemas graves que plantean estos dos documentos estriba en la disparidad de las fechas. Aunque no se han comprobado todas y cada una de las señaladas por Sanchis Sivera en su libro La Catedral de Valencia, en el cap. dedicado a los órganos, es difícil pensar en una equivocación. Sin embargo los documentos que se aportan atribuidos al mismo Fray Antonio Llorens y Fray Juan Olius son anteriores a la renovación del instrumento. ¿Dónde está la explicación? Sanchis Sivera señala el año 1632 como fecha de iniciación de los trabajos de los PP. Franciscanos, fecha confirmada, además, por el Llibre d'Antiquitats cuando afirma que:

«A 20 de gener de 1632, se comensà a desfer lo orgue gran de la Seu... lo molt Ille Capitol donà orde se demanàs al General De Sent Francés licencia per a que dexàs venir a Valencia per a fer dita obra a tres pares del orden de Sent Francés que estaven en Barcelona... lo major y mes gran oficial frare Antony Lorens, prévere, lo segón... frare Juan Olips, prevere, y lo tercer frare Jaume Vergoñós, lo qual se comensà a ordenar in sacris en Valencia...».

Sin embargo el nuevo documento, que lleva los nombres de Antoni Llorens y Juan Olius, es de 1627. ¿Se equivocaron al escribir la tabla y el pergamino? ¿Se equivocó el copista? o, sencillamente, ¿los dos documentos fueron escritos por los franciscanos en visita antecedente al trabajo que ellos mismos debían realizar? Sea como fuere la realidad de todo ello, lo cierto es que el pergamino 90.241 nos da la composición del órgano grande de la Catedral de Valencia, aunque no de forma tan minuciosa como la escribió Fray A. Llorens sobre el órgano de la Catedral de Lérida, con la cual no es nada sencillo, al menos para mi, llegar a aquilatar el número de juegos reales que contenía éste órgano.

Pese a todo ello, sabemos que «l'orgue gran» de Valencia contaba con 32 registros distribuidos en dos teclados; de éstos, los pertenecientes al gran órgano eran partidos y los nueve de la cadireta - casi con seguridad- eran ¿enteros? puesto que todos y cada uno de ellos dependía solamente de un solo registro, ya que dice el pergamino: «La cadireta tiene nueve registros todos a la mano derecha». La costumbre generalizada era colocar los registros de mano derecha al lado derecho del teclado y los de la izquierda al lado izquierdo, y, precisamente en ello tenemos una primera dificultad de intelección, porque difícilmente podemos decir, por los solos documentos, si era o no partida la cadereta aunque luego necesitemos que fuera de ese tipo. 
La segunda dificultad nos la plantea el segundo documento que presentamos, porque se ignora la composición del órgano segundo, tal cual indica la tabla, ni a qué instrumento se refiere el franciscano. No se, si aun encontrando el contrato del Cabildo con los PP. Franciscanos para la construcción del órgano tendríamos tantos pormenores como los que indica el contrato de Lérida. Ningún contrato valentino es tan explicito como ese...

El «modo de registrar el segundo órgano desta Sancta Iglesia de Valencia» no debe referirse a la cadireta dado que el primer documento nos habla del «modo de registrar el presente órgano grande y su cadireta». Necesariamente debiera hacer referencia al órgano pequeño - l'orgue chic - o segundo órgano de la Catedral colocado frente a frente del órgano grande aunque para ello necesitemos que éste instrumento sea también partido.

Este instrumento era muy pequeño y, a juzgar por documentos posteriores, solamente se completó, cuando en 1855 se firmó un contrato entre el Director de la Capilla de Música —que no maestro de capilla - canónigo F. Villalba, y el constructor Miguel Alcarria para reparar «el órgano pequeño», que "no presenta hoy señales de haber sido desmontado después de su primitiva colocación»... «con adición de cuatrocientos doce tubos de música y demás variaciones»*.

Según Alcarria la contextura de este órgano era la siguiente:

Flautado mayor en ambas manos

Octava general, idem

Tapadillo general, idem

Docena y quincena

Nazardo en duodécima

Nazardo en décima quinta

Quincena y vigésima segunda

Címbala de tres tubos por marcha

Dos juegos de contras de doce palmos
47 tubos

$$
\begin{aligned}
& 94 \text { tubos } \\
& 47 \text { tubos } \\
& 47 \text { tubos } \\
& 94 \text { tubos } \\
& 147 \text { tubos } \\
& 16 \text { tubos }
\end{aligned}
$$

Alcarria haría el teclado nuevo, añadiendo el «Do y Re graves sostenidos en la mano izquierda; Do sostenido y Re natural en la derecha». También añadiría la

$\begin{array}{ll}\text { Corneta magna partida de mano derecha, de } 8 \text { tubos por marcha } & 208 \\ \text { Flauta travesera de dos tubos por marcha, partido de mano derecha } & 52 \\ \text { Trompa real partido de mano izquierda } & 25 \\ \text { Clarín en quince partido de mano izquierda } & 25 \\ \text { Trompa magna partido en mano derecha } & 26 \\ \text { Clarín claro partido idem } & 26 \\ \text { Nazardo en décima séptima partido de mano izquierda } & 25 \\ \text { Nazardo en décima nona, partido de mano izquierda } & 25 \\ & \\ \text { Total cuatrocientos doce } & 412\end{array}$

* Miguel Alcarria es el único organero que se preocupó de dar a conocer, en forma detallada y minuciosa, incluso editando un folleto, las reformas realizadas. 
Con todo ello el órgano de la Catedral contaba con 17 juegos; pero ello ya en el siglo XIX.

No es fácilmente asumible que el «modo de registrar el segundo órgano» se refiera al órgano pequeño que solamente contaba con 9 juegos, mientras que en ese modo de registrar se hace frecuente alusión al registro 10 .

He ahí una de las incógnitas que, al menos yo, no veo de fácil solución. También cabría que ese segundo órgano siga refiriéndose al órgano grande, admitiendo una confusión en el copista del original que redactaría A. Llorens.

Pese a estas dificultades, creo que los dos documentos son interesantes y aportan nueva luz al conocimiento del órgano de Valencia.

El modo que se ha de tener en registrar el presente órgano

Grande y su cadireta desta Sancta Iglesia Mayor de Valencia es el siguiente. Año de 1627.

Primo en dicho Organo hay diecisiete Registros, los cuales se señalan con esta figura redonda $\mathrm{O}$ porque ansi están ellos: los nueve a la mano derecha del tañedor, y los ocho a la mano izquierda. Mas hay otro registro pequeño a la mano izquierda más alto un poco de los otros el qual es de las Contras mayores.

Mas hay otros tres Registros a la misma [mano] más bajos que estos son los contrabaxos de la cadireta.

Comiença la composición de los diecisiete Registros y el número dellos por el más baxo de la mano derecha, que es el principal y va subiendo hacia arriba y bajando con su número de 1.2.3. hasta 17.

Mas va también la declaración de las mixturas y diferencias más principales que hay, las cuales son el lleno, flautados y Nazares: y con estas se hacen otras muchas diferencias: y la misma composición tiene la cadireta $8^{\mathrm{a}}$ arriba.

Los registros de los Nazares son cuatro, los quales se señalan con una + cruz: estos han de estar tirados para fuera, quando se tañe el lleno: aunque la dozena gorda que es el 13 Registro bien puede ir alguna vez con el lleno, para darle más cuerpo. Todos los registros puestos para dentro tañen. Ase de advertir que los tres registros de las Contras de la Cadireta van al revés porque puestos para dentro no tañen: los quales registros que son todos veinte y uno son estos que se siguen.

Registros de la mano izquierda
$\begin{array}{lll}14 & 0 & \text { Contras mayores } \\ +15 & 0 & \text { Lleno } \\ 16 & 0 & \text { Lleno } \\ 17 & 0 & \text { Lleno }\end{array}$

130 dozena gorda + Nazard

120 flauta pequeña

11. 0+ Nazard flautado de la esperanza

100 flauta gorda

Registros de la mano derecha

$\begin{array}{lll}6 & 0 & \text { lleno } \\ 7 . & 0 & \text { lleno } \\ +8 & 0 & \text { Nazar. } \\ 9 & 0 & \text { lleno }\end{array}$

Mas hay estos tres Registros los cuales son de las contras

$\begin{array}{lll}5 & 0 & \text { lleno } \\ 4 & 0 & \text { lleno } \\ 3 & 0 & \text { Flautado } 3^{\circ} \\ 2 & 0 & \text { Flautado } 2^{\circ} \text { en octava } \\ 1 & 0 & \text { Flautado de la cara } \\ & & \\ 3 & 0 & \\ 2 & 0 & \\ 1 & 0 & \end{array}$


de la Cadireta y el $3^{\circ}$ destos es el Nazar de la Cadireta que es el cuarto Registro de la dicha Cadireta: y el $2^{\circ}$ es de la flauta pequeña que es el $3^{\circ}$ Registro de la Cadireta. El primero registro destos no tiene Contras que ya las lleva consigo que es el $2^{\circ}$ de la Cadireta.

El lleno del Organo es todos los registros para dentro, y los cuatro Nazares para fuera: y alguna vez puede ir con el lleno la dozena gorda que es el $13^{\circ}$ Registro para dar mas cuerpo al lleno.

Los flautados son seis, los tres estan a la mano derecha y los otros tres a la mano izquierda: 10 y 12 Registros son flautas, y todas juntas hazen un flautado grande; y cada uno de por si puede tañer: y el mejor flautado son las dos flautas juntas: que son flauta gorda décima, y la pequeña duodécima Nazar.

Flautas naturales son dos Registros que están a la mano izquierda; el uno la flauta gorda 100 y la otra flauta pequeña 120.

El flautado de la Esperanza tan nombrado en esta Iglesia, quanto bueno: y el de la cara, que son del Organo viejo es el undécimo 0 está entre las dos flautas gordas y pequeña viene bien con todos; y a solas es muy bueno y suave.

De las donçaynas

Las Donçaynas son dos órdenes unas mayores en unisonus y otras menores en octava: las unas y las otras vienen bien con qualquier Registro y las dos juntas parecen bien y las grandes con el 100 Registro parecen vigüelas de arco.

Los Registros destas son dos, están a la mano derecha del tañedor encima de los nueve Registros salen por entre el rejado de las dichas Donçaynas.

Registros de la cadireta

La Cadireta tiene nueve registros todos a la mano derecha, aunque al reves, porque comiençan de la cara de dicha Cadireta la qual tiene el tañedor a las espaldas.

El orden y asiento que tienen estos Registros es desta manera, que comiença el primero a las espaldas del tañedor y viene para fuera desta manera.

9.8.7.6.5.4.3.2.1.La cara

Todos hacia el altar Mayor tañen, y tirados hacia el tañedor no.

El lleno desta Cadireta es este los seis Registros que son 1.2.5.7.8.9. hacia el Altar y el 3.4. y 6. hacia el tañedor.

Los flautados son 3 , el primero que es de la cara; el $2^{\circ}$ que es la flauta natural mayor, y esta puede ir con el Nazar para hacerle más abultado.

El $3^{\circ}$ es la flauta pequeña. Todos estos tres flautados se pueden tañer juntos y cada uno de por sí.

Primero Flautado de la cara $0 \quad 1$

Segundo flautado flauta gorda ....................... $0 \quad 2$

Tercero flautado flauta chica ............................ $0 \quad 9$

Con estos y con cualquier dellos puede hacer el tañedor las mixturas que quisiere; y los tres Registros de las contras, que están a la mano izquierda bajo de los del Organo grande son, que el más bajo es deste segundo Registro, que es la flauta gorda.

El segundo es del $3^{\circ}$ destos que es de la flauta pequeña: y el $3^{\circ}$ es del cuarto Registro destos, que es el Nazar. 
De los unisonus

Los unísonus son muchos de la Cadireta, con el Organo grande.

El flautado primero de la cara de la Cadireta con el $2^{\circ}$ del Organo grande.

El $2^{\circ}$ registro de la Cadireta con el 10 del grande.

El flautado pequeño de la cadireta con el 12 del grande.

El primero y $3^{\circ}$ de la cadireta con el $3^{\circ}$ y 15 del grande.

De la unisonus de los Nazares

La flauta gorda de la Cadireta y el $4^{\circ}$ y $6^{\circ}$ con el 10,13 y 15 del grande. Unisonus Nazar menor

El $3^{\circ}$ Registro 4 y 6 de la cadireta con $8^{\circ}, 12$ y 15 del grande

Tercero Unisonus del Nazar

El segundo y $6^{\circ}$ de la Cadireta con el 10 y 15 del grande.

Todos los Registros del Organo y Cadireta son 30 sin los de las donçaynas; los 7 puntos mas bajos de las donçaynas se pueden tañer con los pies con las contras y parecen bajón.

(Aunque no se da la composición de la cadereta, precisamente por los unisonos se deduce perfectamente que con nueve juegos tiene tres de flautas y flautados, así como varios nazardos, y en los otros algún lleno.)

El $2^{\circ}$ documento no tiene signatura y en lugar de estar escrito en pergamino como el anterior, está escrito en papel, pegado posteriormente a unos cartones.

Modo de registrar el segundo organo desta Sancta Iglesia de Valencia

Adviértase primero que para tañer el Organo sin partidos han de tirar los Registros hacia fuera por la parte de mano izquierda: $Y$ abrirse han también los de mano derecha y para que hagan buen efecto ayudarán también con la mano derecha a tirar los de esa misma parte para que salgan bien y lo mismo harán cuando los metieren hacia dentro: y de esta manera saldrán las diferencias bien como están apuntadas en este Arancel.

Organo Ileno. - NUMEROS- 1.2.4.9.10..- Otro: 1.2.5.8.9.10.- Otro: 2.3.9.10. Otro. 1.2.3.8.9.10. Otro.- 2.3.8.9.-Otro- 1.5.6.8.10..- A todos los cuales podrán poner las peanas primeras. -

Flautados: NUMEROS.- 1. Para cantar una voz a solas. Otro.- 1.2. y este para cuando cantaran a dos coros. Otro.- 3..- Otro. 6.- Otro. 2.3. Otro. 2. Otro.- 1.3..- Otro. 1.6. ,-Otro, 3.6. Otro. 1.2.3. Otro.- 2.3.6. Otro. 1.3.6.. A todos estos pueden meter el temblante si quisieren; salvo cuando se canta con el órgano que no puede andar el temblante.

Nazardos.- NUMEROS.- 1.4. Otro.-1.7. Otro. 3.4.7. Otro. 1.2.4. Otro 1.2.4.7. Otro 2.4.7. Otro 3.6.7. Otro 4.6.7. Otro 6.7. Otro 1.2.4.6.7. Otro 2.7. Otro 4.6. Otro 3.4.6.7.. A todos estos pueden meter para los pies el Registro de las peanas número 2.

Diferencias.- NUMEROS. 1.2.5. Otra. 2.3.9. Otra 1.6.8. Otra 1.2.3.9. Otra 1.2.3.5.9. Otra 2.3.5.10. A todas estas pueden poner el registro de las peanas que quisieren.

Cascabeles. NUMEROS. 1.10. Otros 3.10. Otros 1.9. Todos tres con temblante y con el segundo registro de las peanas.

Unísonus. NUMEROS. 1 de la mano derecha.- 2 de mano izquierda. Otros 3 de mano 
derecha. 6 de mano izquierda. Otros 1.3. de mano derecha. 2.6. de mano izquierda. Estos tres unisonus han de ser acompañadas con las peanas del número 2.

Partidos de mano izquieda

Baxones. NUMEROS. 6 de mano derecha.- 1.2.3.4.6.7. de mano izquierda.

Otros. 6 de mano derecha.- 3.4.6.7. de mano izquierda. Otros. 2. de mano derecha- 2.3.4.7. de mano izquierda. Otros. 6 de mano derecha.- 4.6.7. de mano izquierda, hanse de tañer sin peana todas estas cuatro diferencias.

Otros partidos.- NUMEROS.- 1 de mano derecha.- 1.2.4. de mano izquierda. Otros 1.2. de mano derecha- 1.2.5.9. de mano izquierda. Otros 2.3. de mano derecha.- 2.3.5.8. de mano izquierda. Otros. 3.6. de mano derecha. 3.4.6.8. de mano izquierda. Otros 1.2. de mano derecha2.3.8.10. de mano izquierda. Estas cinco diferencias se han de tañer sin las peanas.

Partidos de mano derecha

Cornetilla. NUMEROS.- 6. de mano izquierda. 2 de mano derecha. con temblante y de espacio: Ha de llevar con los pies las peanas del registro 2.

Pajarillos. NUMEROS 6 de mano izquierda.- 3.7. de mano derecha. Otros. 3.6. de mano izquierda.- 4.6.7. de mano derecha. Otros. 6 de mano izquierda.- 6.7. de mano derecha. Otros. 2.3. de mano izquierda.- 3.4.6.7. de mano derecha. A todas estas cuatro diferencias han de poner el Registro 2 de las peanas.

Gaytillas. MUMEROS.- 1.2. de mano izquierda. 2.5.9. de mano derecha Otra. 1.2. de mano izquierda. 1.2.5. de mano derecha. Otra. 1.2. de mano izquierda. 2.3.10. de mano derecha. Otra. 2.6. de mano izquierda. 6.9. de mano derecha. Otra 1.6. de mano izquierda. 2.10. de mano derecha. Otra. 2.6. de mano izquierda, 4.6. de mano derecha. En estos seis partidos han de poner las peanas número 1 .

Tolosana. NUMEROS. 1.2. de mano izquierda. 2.3.8. de mano derecha. Otro. 2.3. de mano izquierda, 6.8. de mano derecha. Con las peanas del segundo registro.

F. AN LLORENZ Y FRAY JUAN OLIUS.

Estos son los dos nuevos documentos que conocemos del órgano catedralicio valenciano. Ciertamente no está todo resuelto. Sin embargo ya sabemos, con certeza, que se trataba de un órgano partido; que, contra lo que pudiera imaginarse, no era rico en lengüetería puesto que ninguna consta, excepción de las Dolçainas. Los grandes registros de lengüeta se irán añadiendo, poco a poco. Así es sabido que en 1693 Roque Blasco añade un juego entero de trompas reales y medio registro de clarín de mano derecha. El gran uso de los partidos con todo tipo de combinaciones que viene a desmentir toda una corriente actual, ciertamente no fundada en la realidad clásica, que es el uso del trémolo, hasta hecho desaparecer en la construcción de nuevos órganos. También se trataba de un instrumento rico en Nazardos y, además, en Llenos.

Es curioso advertir las indicaciones de sonoridad que señala el constructor: Las donçainas con la flauta de 8' parecen vigüelas; las donçainas van bien con cualquier registro, etc. Merecen primer plano los Llenos y la cantidad de combinaciones para tocar partidos a los que hay que sumarles el pedal y el trémolo, de forma especial en los partidos de mano derecha: cornetilla, paxarillos, gaitillas, tolosana. 


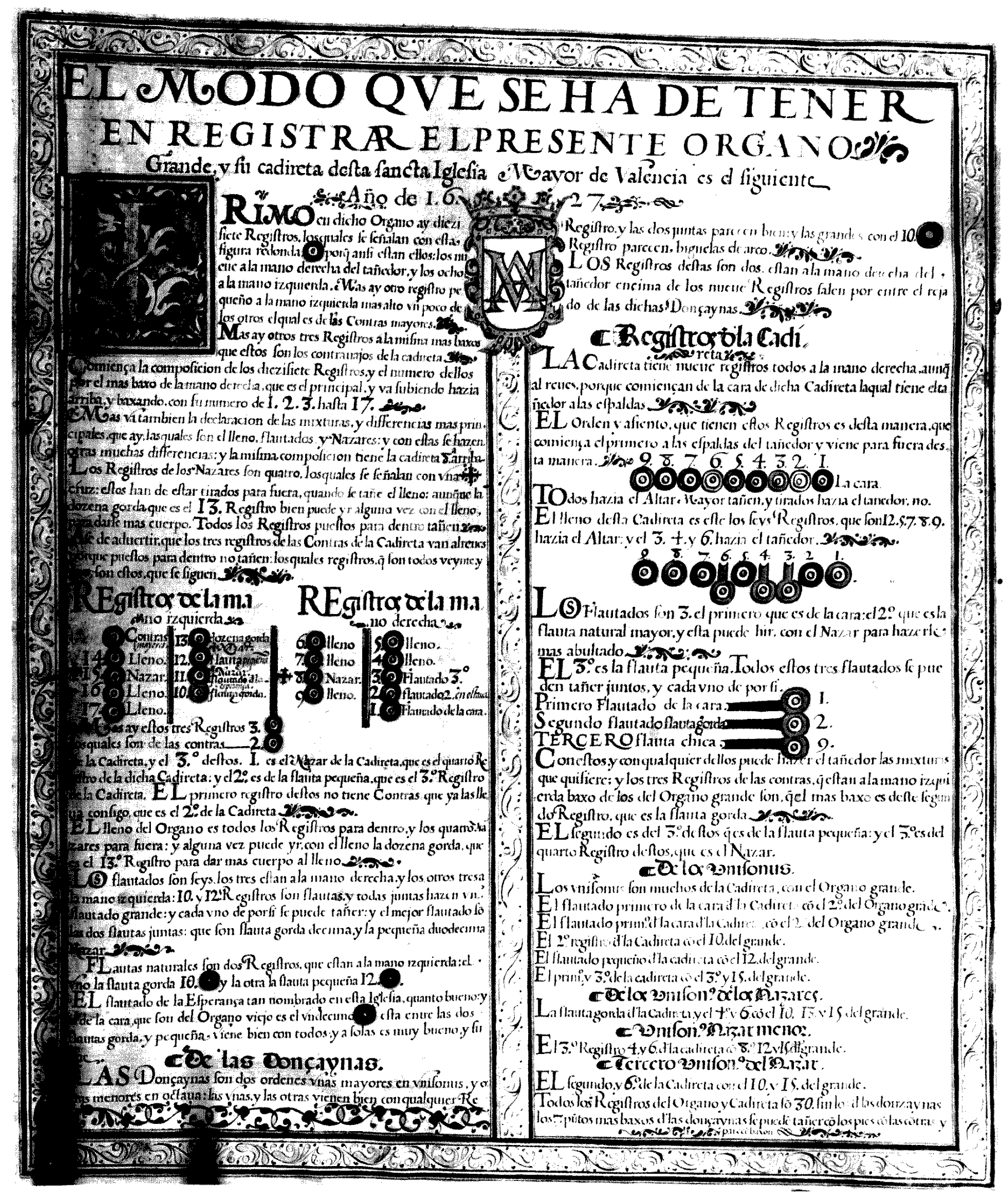

Archivo de la Catedral de Valencia. Pergamino 90.241. Año 1627. 


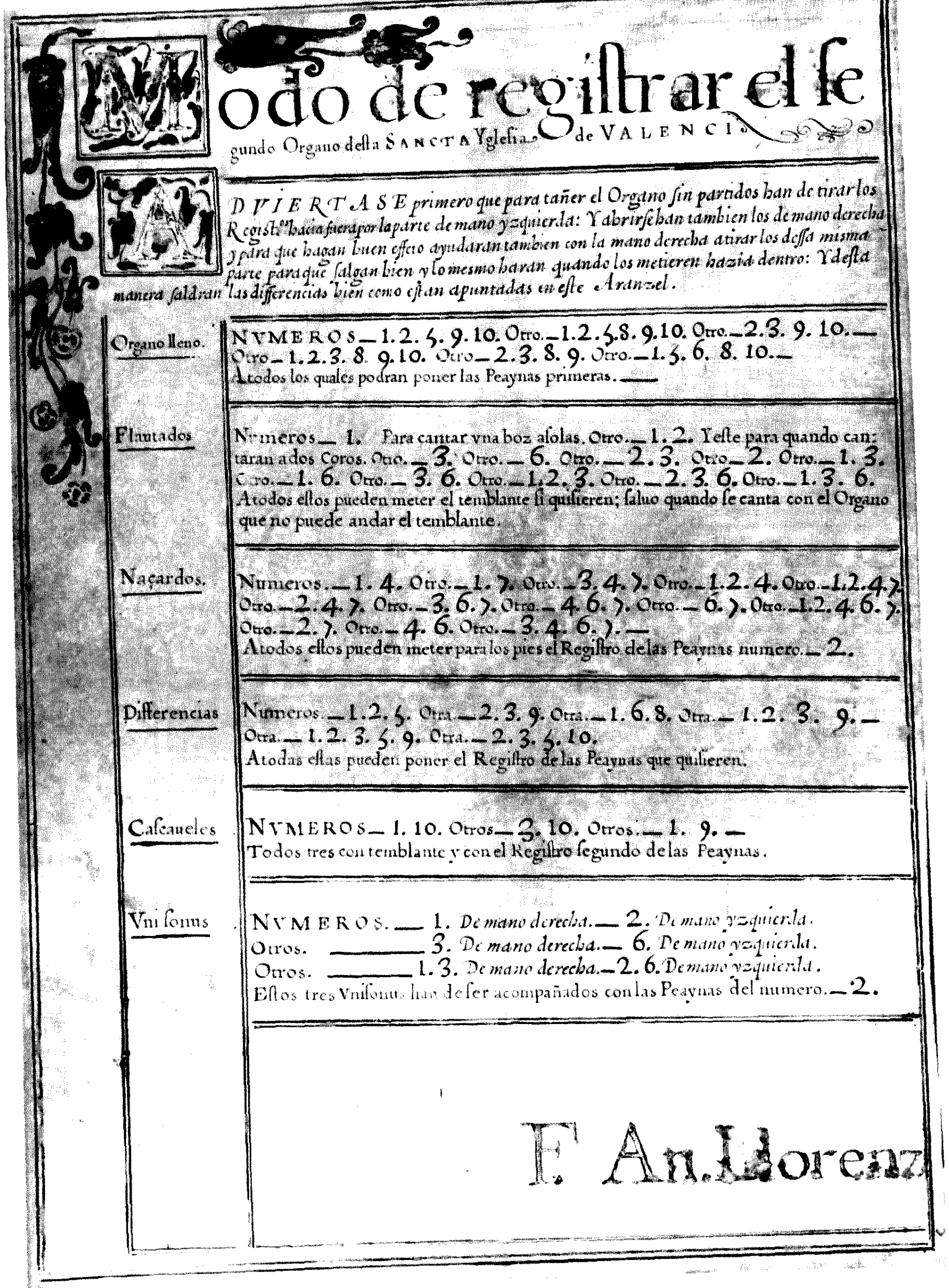

Órgano de la Catedral de Valencia. Tabla de registración. Año 1627. 


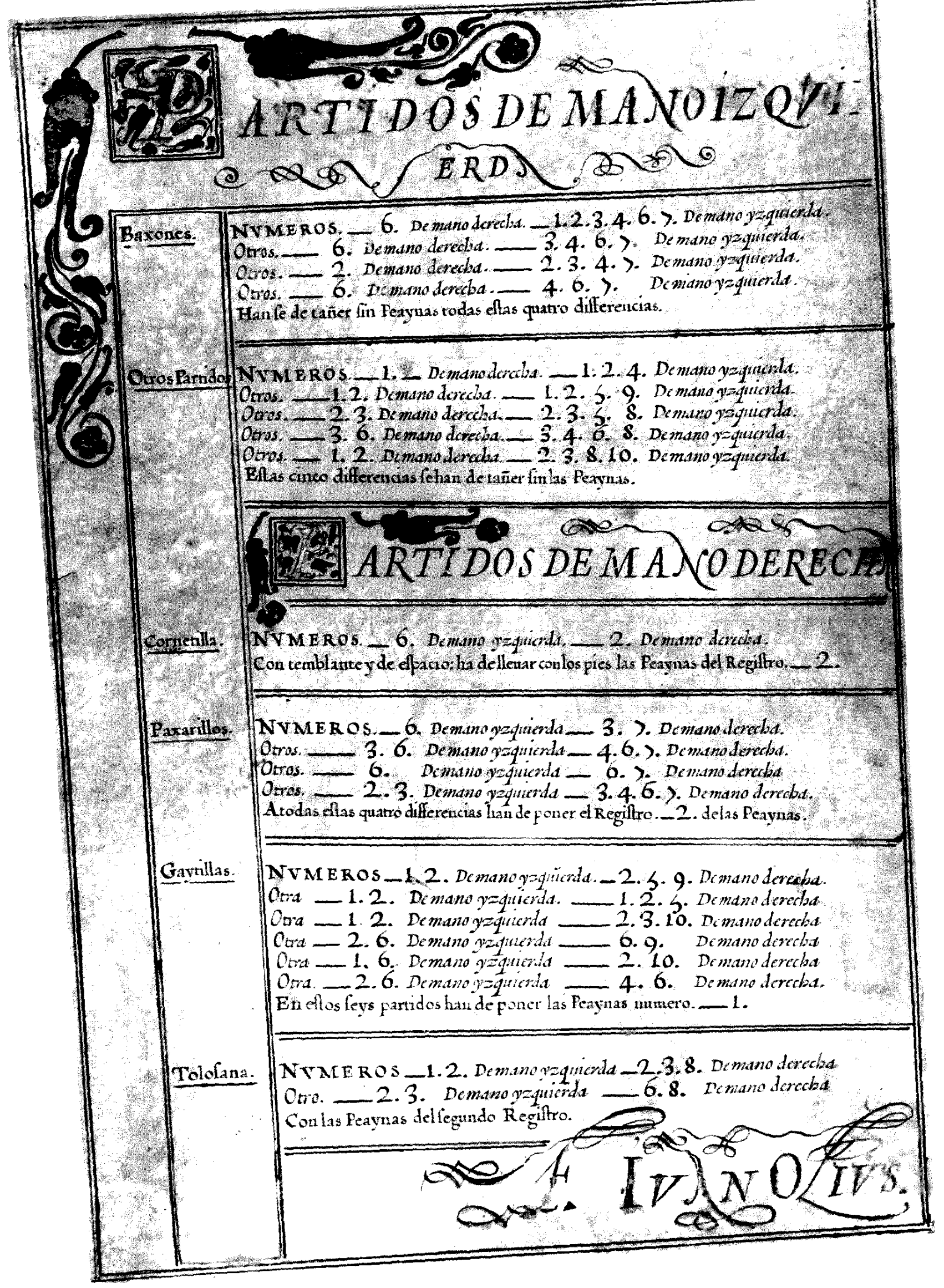

Órgano de la Catedral de Valencia. Tabla de registración. Año 1627. 


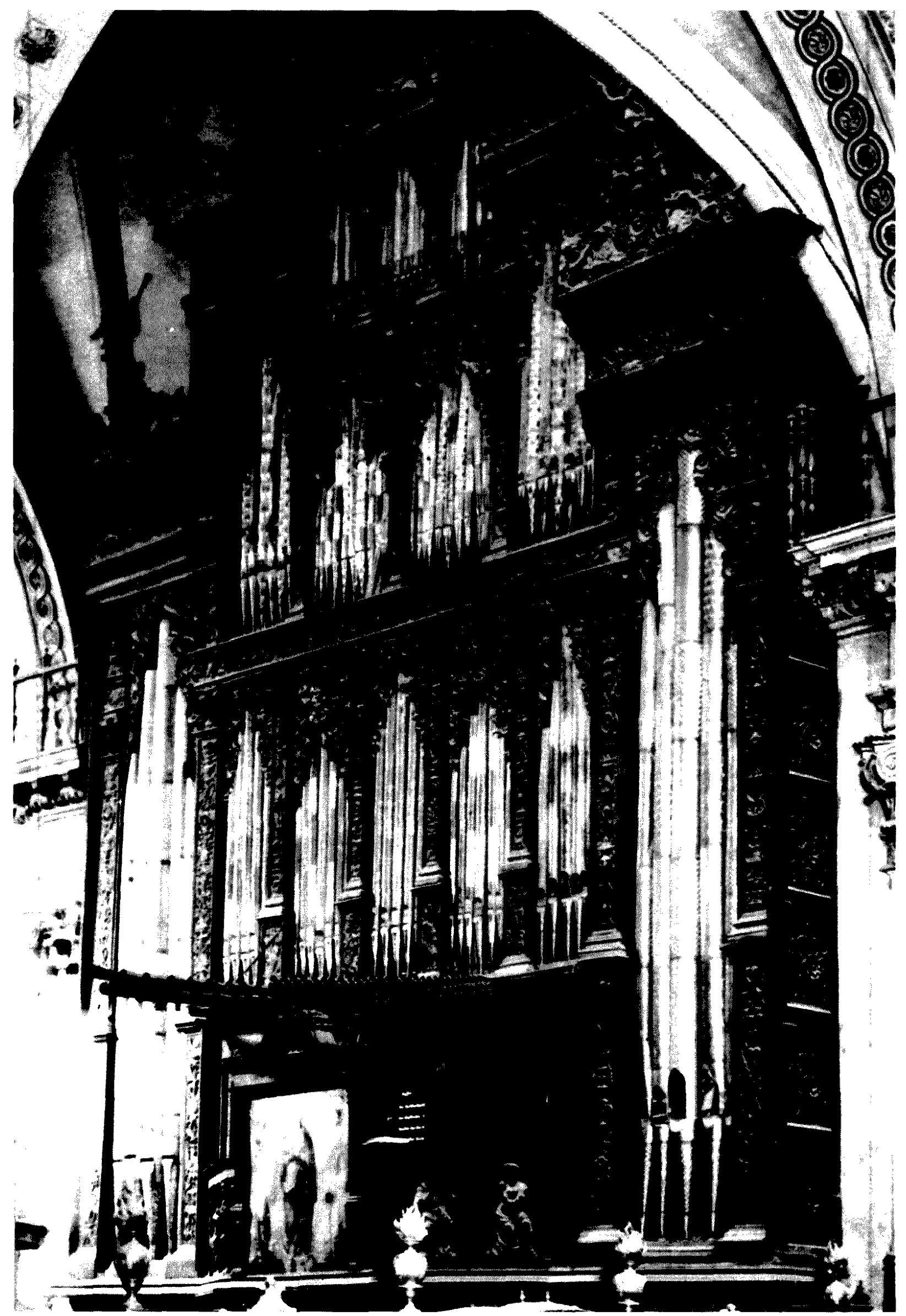

Órgano Mayor de la Catedral de Valencia destruido en 1936. Fue una de las obras maestras valencianas del Renacimiento 